\title{
Relationship Between Mean Platelet Volume-to-Platelet Count Ratio and the Presence and Severity of Coronary Artery Ectasia
}

\author{
Ramazan Asoğlu (iD ${ }^{1, *}$ and Emin Asoğlu ${ }^{2}$ \\ ${ }^{1}$ Cardiology Department, Adıyaman University Training and Research Hospital, Adıyaman, Turkey \\ ${ }^{2}$ Cardiology Department, Mardin Community Hospital, Mardin, Turkey
}

*Corresponding author: Dr. Ramazan ASOĞLU, Cardiology Department, Adıyaman University Training and Research Hospital, Adıyaman, Turkey, Tel: +90-530-776-37-12, Fax: +90-416-223-38-42

\begin{abstract}
Background: Atherosclerosis plays an essential role in the etiopathogenesis of coronary artery ectasia (CAE). The mean platelet volume/platelet count (MPV/PLT) ratio is a new biomarker, and increased MPV/PLT ratio was a predictor of cardiovascular mortality in patients with the acute coronary syndrome. We aimed to assess the association between the CAE and MPV/PLT ratio.

Material and method: The present study included 50 patients with CAE and 45 healthy subjects prospectively. The CAE was defined as dilation of the coronary artery $>1.5$-fold the diameter of the adjacent normal coronary vessels The severity of isolated CAE was determined according to the Markis classification. Information regarding blood tests of the patients was obtained during hospitalization.

Results: MPV/PLT ratio was significantly higher in patients with CAE than in those with normal coronary artery. There was no significant difference between the CAE types regarding MPV/PLT ratio. There was a positive and significant correlation between the CAE and MPV/PLT ratio $(r=0.364$, $\mathrm{p}=0.001)$.

Conclusion: MPV/PLT ratio is significantly higher in patients with isolated CAE when compared to controls with normal coronary artery. MPV/PLT ratio is significantly correlated with the presence of CAE.
\end{abstract}

\section{Keywords}

Coronary artery ectasia, Coronary artery disease, Mean platelet volume, Mean platelet volume-to-platelet count ratio

\section{Introduction}

Coronary artery ectasia (CAE) is defined as the diameter of the ectatic segment being at least 1.5 times larger compared to an adjacent healthy segment [1]. CAE has been observed in around $1 \%$ to $5 \%$ of the patients in the coronary angiography units [2]. Up to $20 \%$ of CAE cases are considered congenital, and $10 \%$ to $20 \%$ of CAE are associated with inflammatory diseases or might be related to inflammatory activities [3]. CAE is generally coexisting with atherosclerotic coronary artery disease (CAD) [4], which is the primary cause of CAE, and it shares similar histopathological characteristics of low-grade vascular inflammation [5]. A previous study suggested that severe inflammation could be involved in the pathogenesis of CAE [6]. Also, CAE has some characteristics such as impaired coronary flow, delayed coronary filling with contrast media, and retention of contrast media within dilated coronary segments $[7,8]$. Isolated diffuse CAE may be related to reduced coronary flow reserve and myocardial perfusion abnormalities $[9,10]$. Moreover, thrombus formation with distal embolization and vasospasm are the most severe consequence of CAE [11].

One important factor associated with Coronary artery disease (CAD) is platelet activation and increased platelet numbers can be a sign of inflammation. Platelets with a large volume have a more thrombotic potential [12] and increased mean platelet volume (MPV) is an independent predictor of a massive intracoronary thrombus in acute myocardial infarction [13]. Increased MPV has a more significant effect on hemostasis, and it is metabolically and enzymatically associated with more active platelets [12]. MPV, an indicator of platelet re-

Citation: Asoğlu R, Asoğlu E (2019) Relationship between Mean Platelet Volume-to-Platelet Count Ratio and the Presence and Severity of Coronary Artery Ectasia. Int Arch Cardiovasc Dis 3:024. doi. org/10.23937/2643-3966/1710024

Accepted: September 26, 2019; Published: September 28, 2019

Copyright: (C) 2019 Asoğlu R, et al. This is an open-access article distributed under the terms of the Creative Commons Attribution License, which permits unrestricted use, distribution, and reproduction in any medium, provided the original author and source are credited. 
activity, is increased in atherosclerotic heart diseases, especially in myocardial infarction [14]. The platelet counts and MPV might be a link in the pathophysiology of diseases predispose to inflammation. The mean platelet volume/platelet count (MPV/PLT) ratio is a new biomarker and increased MPV/PLT ratio was a better predictor of long-term CV mortality in non-STEMI (NSTEMI) patients than MPV and platelet alone [15]. This study aimed to investigate a relatively inexpensive and easily available inflammatory marker, which is MPV/PLT, in patients with CAE.

\section{Materials and Methods}

A total of 95 patients were included in this study after the local Ethics Committee of our hospital approved the study protocol. We prospectively analyzed the electronic patient data recording system of our hospital for the patients between March 2018 and May 2019. A total of 95 patients were included in the study, including 50 patients with isolated CAE and 45 with normal coronary artery. Patients with isolated CAE had non-obstructive epicardial lesions $<20 \%$.

All the groups were evaluated by physical examination and underwent transthoracic echocardiography for analysis of structural heart disease. Patients' laboratory and clinical characteristics were accessed through medical records. Clinical information included data on systemic hypertension (HTN), diabetes mellitus (DM), dyslipidemia, smoking, and previous history of CAD. Diabetes was based on a fasting blood sugar level $\geq 126 \mathrm{mg} / \mathrm{dL}$ or use of an anti-diabetes medication. Hypertension was reported for systolic blood pressure $\geq 140 \mathrm{mmHg}$, diastolic blood pressure $\geq 90 \mathrm{mmHg}$ or use of antihypertensive agents. Hyperlipidemia was reported for total cholesterol $(\mathrm{TC}) \geq 200 \mathrm{mg} / \mathrm{dL}$, low-density lipoprotein (LDL) level $\geq 130 \mathrm{mg} / \mathrm{dL}$ or use of cholesterol-lowering medication. Smoking included active or previous ( $>10$ pack-years) tobacco use. A positive family history of CAD was based on evidence such as a history of acute coronary syndromes in first-degree relatives before 60 years of age. Body mass index (BMI) was calculated by dividing weight in $\mathrm{kg}$ by height in $\mathrm{m}^{2}\left(\mathrm{~kg} / \mathrm{m}^{2}\right)$. 12-lead electrocardiography (ECG) was recorded, and transthoracic echocardiography study was performed with a 3.5-MHz transducer to all patients (Vivid 3; GE Medical System, Horten, Norway). The left ventricular ejection fraction (LVEF) was measured according to Simpson's method [16].

Exclusion criteria were uncontrolled hypertension, anemia, uncontrolled DM, left ventricular dysfunction (left ventricular ejection fraction $<50 \%$ ) or hypertrophy, acute coronary syndromes (ACS), previous coronary artery bypass grafting, a history of percutaneous coronary intervention, valvular heart disease, congenital heart disease, abnormal thyroid function tests, renal or hepatic dysfunction (creatinine $>1.5$ $\mathrm{mg} / \mathrm{dL}$, aspartate aminotransferase and alanine tran- saminase $>2$ the upper limit of normal, respectively), a known malignancy, acute or chronic infection, inflammatory diseases, thrombocytopenia, hemolytic failure, autoimmune or neoplastic disease, and any medication that might interfere with measurement of MPV/PLT ratio.

The indication for coronary angiography was the presence of typical angina or positive or equivocal results of treadmill exercise or myocardial single photon emission computed tomography for myocardial ischemia in both the groups. All patients underwent elective coronary artery angiography using Siemens Axiom Artis DFC (Siemens Medical Solutions, Erlangen, Germany). Coronary angiograms were performed via a femoral approach by using the Judkins technique without the use of nitroglycerin. İohexol (Omnipaque) was used as a contrast agent during coronary angiography. Angiograms were recorded on DICOM digital media (Siemens Medical Solutions, Erlangen, Germany; 25 frames/ms) and were reviewed by two interventional cardiologists who were unaware of the clinical information. The CAE was defined as dilation of the coronary artery $>1.5$-fold the diameter of the adjacent normal coronary vessels [17]. The CAE classification, previously described by Markis, et al. was used [4]. A normal segment was defined as a coronary artery segment without ectasia or stenosis. If there was no identifiable adjacent normal segment, the mean diameter of the corresponding coronary segment in the control group used as normal values. Absence of any atherosclerotic plaques was regarded as a normal coronary artery.

Before coronary angiography, blood samples were taken after 20 minutes of supine rest and the following fasting for 12 hours from all patients for laboratory analysis in standard tubes containing ethylenediaminetetraacetate acid (EDTA). We analyzed the blood samples of all the groups using an automatic blood counter immediately. Urea, creatinine, serum sodium, potassium, TC, HDL, LDL, and triglyceride levels were determined. Hematological parameters, including hemoglobin (HB), white blood cell (WBC) count, platelet count, and MPV, were analyzed by $\mathrm{LH}$ 780 analyzer (Beckman Coulter Inc, Miami, Florida). MPV/PLT ratio was calculated as the ratio of the MPV to the platelet count. The study was conducted by the Helsinki Declaration, and the local ethics committee approved the protocol. All patients and control individuals gave informed consent.

\section{Statistical Analyze}

Data were analyzed with SPSS software version 20.0 for Windows (SPSS Inc, Chicago, Illinois). The Kolmogorov-Smirnov test was used to verify that continuous variables were normally distributed. Normally distributed variables were expressed as mean \pm standard deviation (SD). The categorical variables are 
Table 1: The demographic and blood characteristics of the patients.

\begin{tabular}{|c|c|c|c|}
\hline & $\operatorname{CAE}(n=50)$ & Control $(n=45)$ & $\mathbf{p}$ \\
\hline Age (years) & $50.46 \pm 11.94$ & $47.27 \pm 8.94$ & 0.14 \\
\hline Male \%(n) & $40(20)$ & $48(22)$ & 0.38 \\
\hline Hypertension (\%) n & $36(18)$ & $24(11)$ & 0.22 \\
\hline Hyperlipidemia (\%) n & $6(3)$ & $7(3)$ & 0.89 \\
\hline Diabetes mellitus (\%) $n$ & $20(10)$ & $22(10)$ & 0.79 \\
\hline Smoking (\%) n & $34(17)$ & $17(8)$ & 0.07 \\
\hline Family history (CAD) (\%) n & $34(17)$ & $20(9)$ & 0.12 \\
\hline ASA (\%) n & $26(13)$ & $13(6)$ & 0.12 \\
\hline Beta blocker (\%) n & $24(12)$ & $9(4)$ & 0.04 \\
\hline ACE inhibitor/ARB (\%) n & $38(19)$ & $22(10)$ & 0.09 \\
\hline CCB $(\%) n$ & $14(7)$ & $4(2)$ & 0.11 \\
\hline Diuretic (\%) n & $36(18)$ & $22(10)$ & 0.14 \\
\hline Statin (\%) $n$ & $12(6)$ & $9(4)$ & 0.62 \\
\hline OAD (\%) n & $20(10)$ & $22(10)$ & 0.79 \\
\hline BMI $\left(\mathrm{kg} / \mathrm{m}^{2}\right)$ & $25.69 \pm 2.47$ & $25.49 \pm 2.60$ & 0.69 \\
\hline Systolic BP (mmHg) & $122.00 \pm 12.41$ & $122.56 \pm 12.37$ & 0.82 \\
\hline Diastolic BP (mmHg) & $76.30 \pm 8.32$ & $77.22 \pm 8.16$ & 0.58 \\
\hline Ejection Fraction (\%) & $56.82 \pm 3.77$ & $57.22 \pm 3.62$ & 0.59 \\
\hline Heart rate (bpm) & $77.80 \pm 12.21$ & $82.44 \pm 13.64$ & 0.08 \\
\hline Serum glucose (mg/dl) & $115.24 \pm 32.16$ & $104.44 \pm 28.57$ & 0.08 \\
\hline Creatinine (mg/dl) & $0.77 \pm 0.15$ & $0.72 \pm 0.16$ & 0.11 \\
\hline Total cholesterol (mg/dl) & $186.88 \pm 39.47$ & $176.53 \pm 51.37$ & 0.27 \\
\hline Triglyceride (mg/dl) & $186.72 \pm 51.77$ & $180.11 \pm 55.80$ & 0.55 \\
\hline High density lipoprotein (mg/dl) & $42.06 \pm 11.92$ & $44.18 \pm 12.78$ & 0.42 \\
\hline Low density lipoprotein (mg/dl) & $114.10 \pm 31.25$ & $117.22 \pm 31.21$ & 0.62 \\
\hline White blood cell count $\left(10^{3} / \mathrm{mm}^{3}\right)$ & $8.04 \pm 1.82$ & $7.27 \pm 1.92$ & 0.04 \\
\hline Hemoglobin (g/dL) & $13.90 \pm 1.83$ & $13.33 \pm 1.91$ & 0.14 \\
\hline Hematocrit (\%) & $42.84 \pm 4.89$ & $41.69 \pm 5.24$ & 0.27 \\
\hline Platelet count $\left(10^{3} / \mathrm{mm}^{3}\right)$ & $252.94 \pm 46.59$ & $264.78 \pm 36.71$ & 0.17 \\
\hline Platelet distribution width, \% & $18.90 \pm 1.90$ & $17.67 \pm 2.32$ & $<0.01$ \\
\hline Plateletcrit, \% & $0.15 \pm 0.06$ & $0.14 \pm 0.05$ & 0.41 \\
\hline Mean platelet volume, fL & $8.22 \pm 1.30$ & $7.20 \pm 0.90$ & $<0.01$ \\
\hline Neutrophil $\left(10^{3} / \mathrm{mm}^{3}\right)$ & $5.36 \pm 2.23$ & $4.36 \pm 1.66$ & 0.01 \\
\hline Lymphocyte $\left(10^{3} / \mathrm{mm}^{3}\right)$ & $2.64 \pm 0.87$ & $2.42 \pm 0.94$ & 0.24 \\
\hline MPV/PLT, n & $0.03 \pm 0.01$ & $0.02 \pm 0.01$ & $<0.01$ \\
\hline
\end{tabular}

CAE: Coronary artery ectasia; CAD: Coronary artery disease; ASA: Acetylsalicylic acid; ACE: Angiotensin Converting Enzyme; ARB: Angiotensin Receptor Blockers; CCB: Calcium Channel Blockers; OAD: Oral Antidiabetic Drug; BMI: Body mass index; MPV/PLT: Mean platelet volume/platelet count.

presented as percentages. Differences between two groups were evaluated with Student's unpaired t-test for parameters with a normal distribution. One-way analysis of variance (ANOVA) test followed by the Tukey post hoc test was used for multiple comparisons. The frequencies of nominal variables were compared using Fisher's exact test or chi-square test. The Spearman test was used for correlation analysis. A Receiver-operating characteristic (ROC) curve analysis was used to determine the optimum cutoff level for the MPV/PLT and Platelet distribution width (PDW) va- lues that best predicted CAE. Statistical significance was defined as $P<0.05$.

\section{Results}

A total of 95 patients were included (50 patients with isolated CAE and 45 with normal coronary artery). The demographic and clinical data of the study population are presented in Table 1 . No difference was found in the demographic characteristics between the groups regarding age and gender. There was no significant difference between the groups regar- 


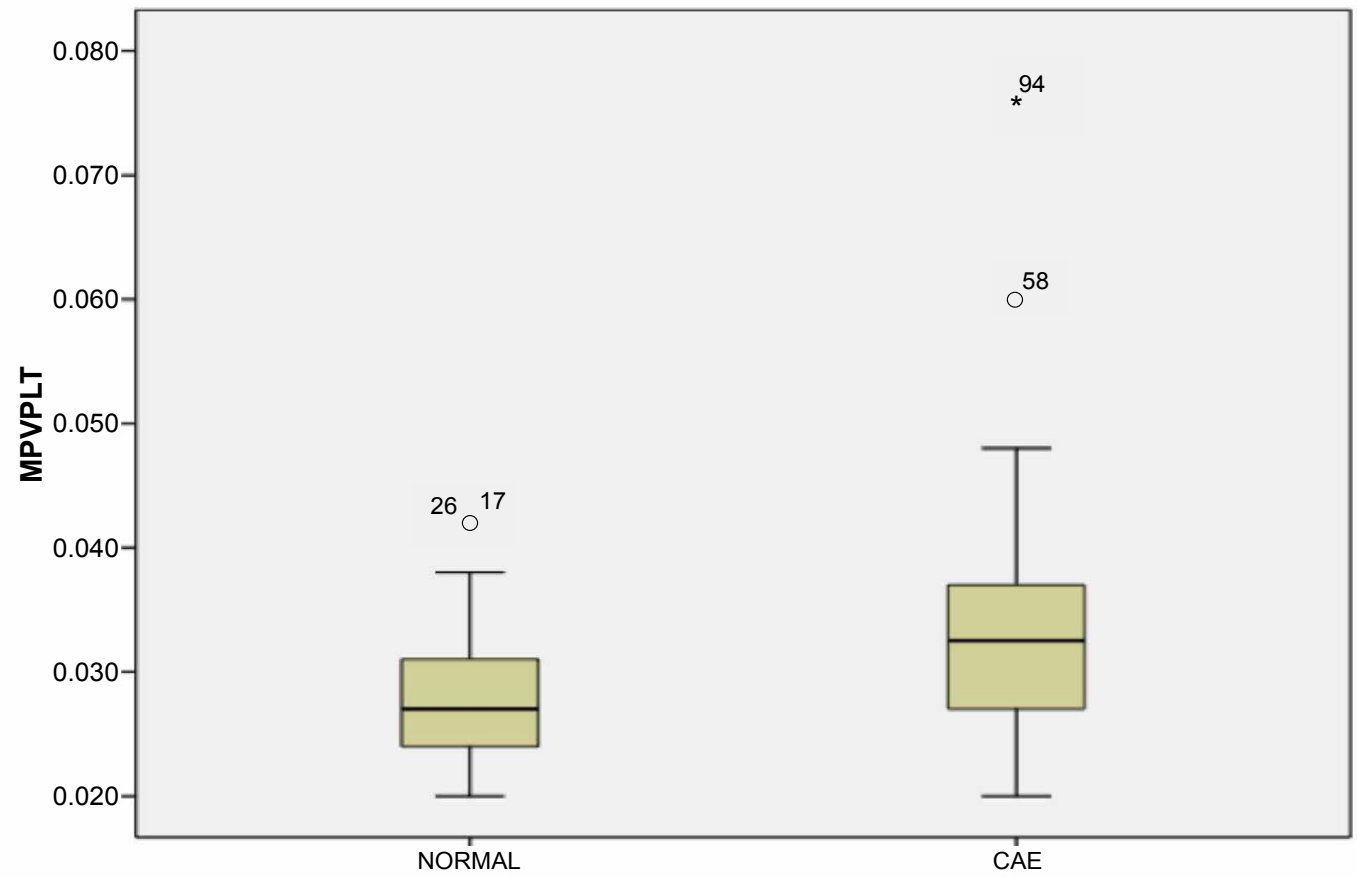

Figure 1: MPV/PLT (Mean platelet volume/platelet) values between CAE (Coronary artery ectasia) and control groups.

Table 2: Number and distribution of coronary vessels affected by ectasia Modified Markis Classification.

\begin{tabular}{|l|l|l|}
\hline & $\%$ & number \\
\hline LAD & 62 & 31 \\
\hline LAD-Focal & 44 & 22 \\
\hline LAD-Diffuse & 18 & 9 \\
\hline CX & 58 & 29 \\
\hline CX-Focal & 26 & 13 \\
\hline CX-Diffuse & 32 & 16 \\
\hline RCA & 28 & 14 \\
\hline RCA-Focal & 12 & 6 \\
\hline RCA-Diffuse & 16 & 8 \\
\hline Left Main & 10 & 5 \\
\hline 1 Vessel & 58 & 29 \\
\hline 2 Vessel & 36 & 18 \\
\hline 3 Vessel & 6 & 3 \\
\hline Type-1 & 16 & 8 \\
\hline Type-2 & 12 & 6 \\
\hline Type-3 & 20 & 10 \\
\hline Type-4 & 52 & 26 \\
\hline
\end{tabular}

LAD: Left anterior descending artery; CX: Circumflex artery; RCA: Right coronary artery.

ding ejection fraction, heart rate, and body mass index. The beta blocker usage is higher in the CAE group. Biochemical parameters were similar between the groups regarding glucose, creatine, total cholesterol, triglyceride, low-density lipoprotein, and high-density lipoprotein, whereas the white blood cell, PDW, neutrophile, and MPV were significantly higher in the CAE group. Against the platelet count was similar, the MPV/PLT ratio was significantly higher in
Table 3: The correlation between coronary artery ectasia and platelet count, platelet distribution width, plateletcrit, mean platelet volume and MPV/PLT ratio.

\begin{tabular}{|l|l|l|}
\hline & r & p \\
\hline Platelet count $\left(10^{3} / \mathrm{mm}^{3}\right)$ & -0.113 & 0.274 \\
\hline Platelet distribution width, \% & 0.301 & 0.003 \\
\hline Plateletcrit, \% & 0.081 & 0.437 \\
\hline Mean platelet volüme, fL & 0.415 & 0.001 \\
\hline MPV/PLT, n & 0.364 & 0.001 \\
\hline
\end{tabular}

MPV/PLT: Mean platelet volume/platelet count.

the CAE group than the control group $(p<0.01)$ (Figure 1). Table 2 shows the number and distribution of coronary vessels affected by ectasia. There was no significant difference between the CAE types (Markis classification) regarding MPV/PLT ratio (Figure 2). There was a positive and significant correlation between the CAE and PDW, MPV, and MPV/PLT ratio (Table 3). Figure 3 presents the correlation between the MPV/PLT and CAE $(r=0.364, p=0.001)$. In ROC curve analyzes, a PDW and MPV/PLT values of 18.50 and 0.027 were determined as an effective cutoff point in coronary artery ectasia with a sensitivity of $74 \%, 66 \%$ and a specificity of $58 \%, 63 \%$, respectively (Figure 4).

\section{Discussion}

This study demonstrated two significant findings in patients with CAE. First, the MPV/PLT ratio, MPV, and PDW values were significantly higher in the CAE group. Second, there was a positive and significant correlation between the CAE and PDW, MPV, and MPV/PLT. These results suggest increased serum MPV/PLT ratio and MPV levels might be related to 


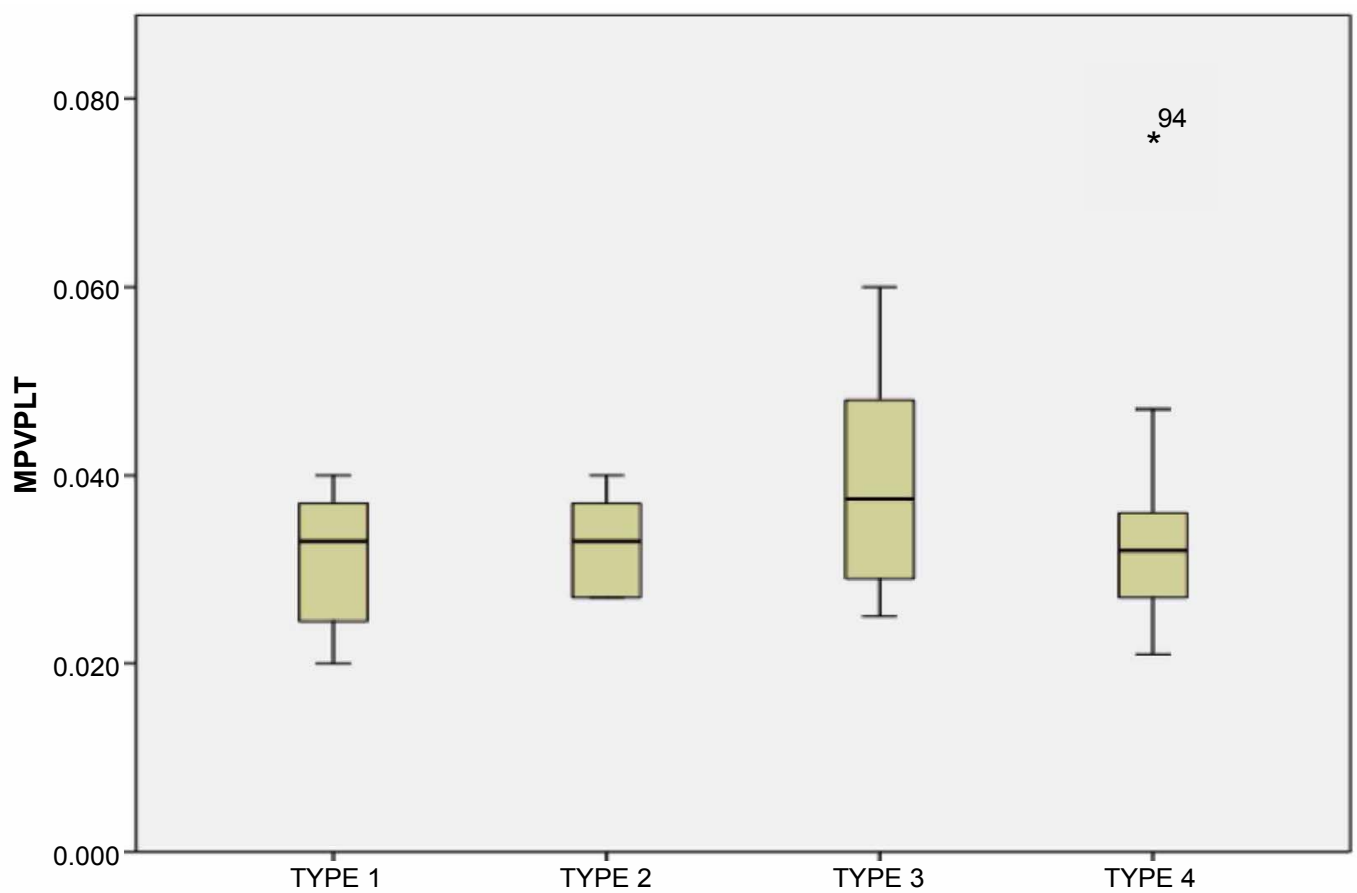

Figure 2: MPV/PLT (Mean platelet volume/platelet) ratios between the coronary artery ectasia types (Markis classification).

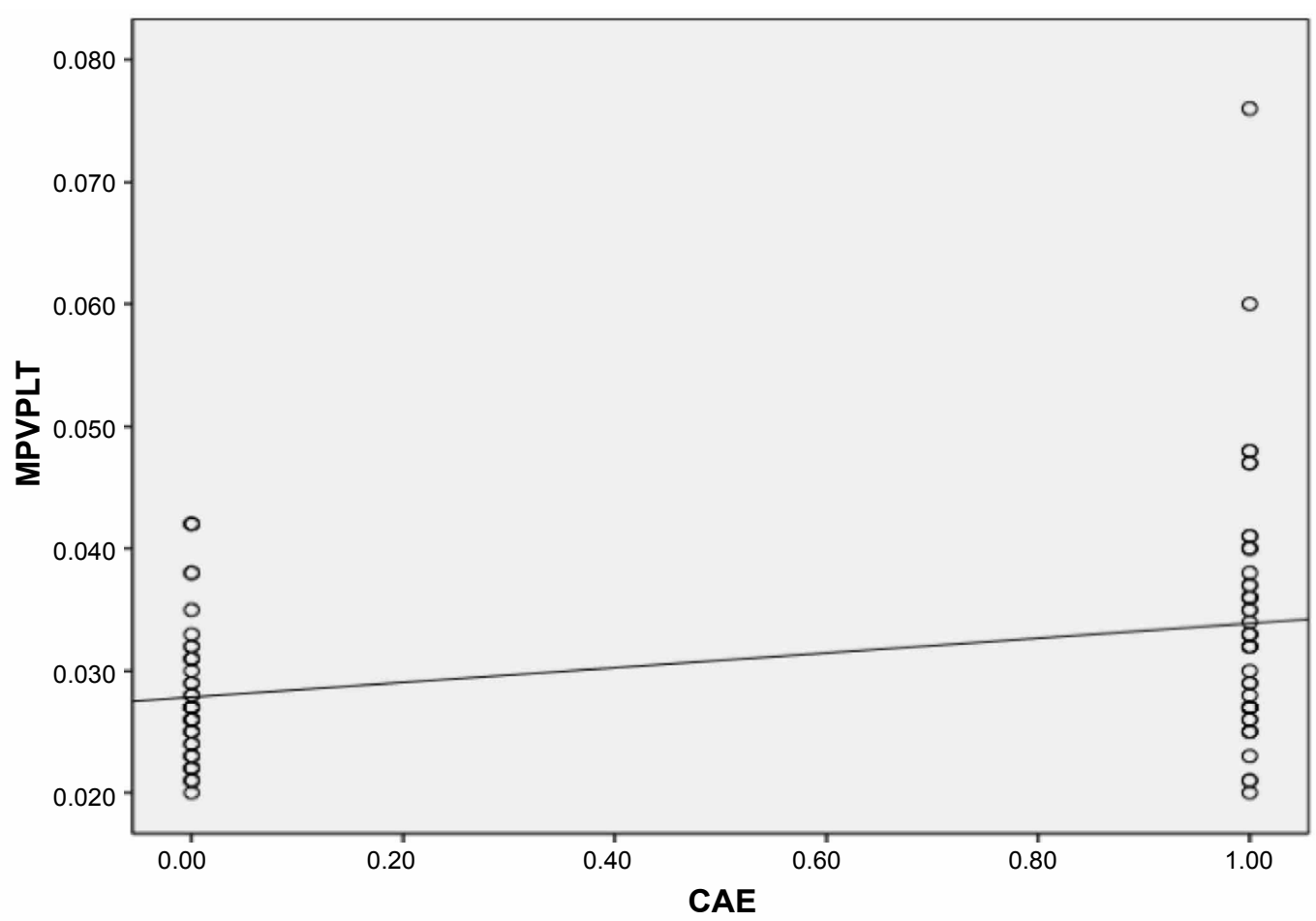

Figure 3: The correlation between MPV/PLT (Mean platelet volume/platelet) ratio and CAE (Coronary artery ectasia).

the inflammation, endothelial dysfunction, and atherosclerotic process of CAE.

MPV/PLT ratio is a novel biomarker used as a prognostic indicator of acute coronary syndromes (ACS). The MPV/PLT ratio may reflect the factors involved in the pathogenesis of ACS more accurately than MPV and platelet separately. Azab, et al. showed that MPV/PLT ratio was associated with four-year all-cause mortality, but no association was obser- ved between MPV and platelet in Non-ST-elevation myocardial infarction patients [15]. Additionally, in a study performed by Bolat, et al. presented that the rate of one-year non-fatal re-infarction was higher in the highest MPV/PLT ratio group compared to middle and lowest groups [18]. In the same study, the authors claimed that the increased MPV/PLT ratio was an indicator of increased platelet activation and aggregation, and this finding can explain the raised post-ST-elevation myocardial infarction re-infarction 


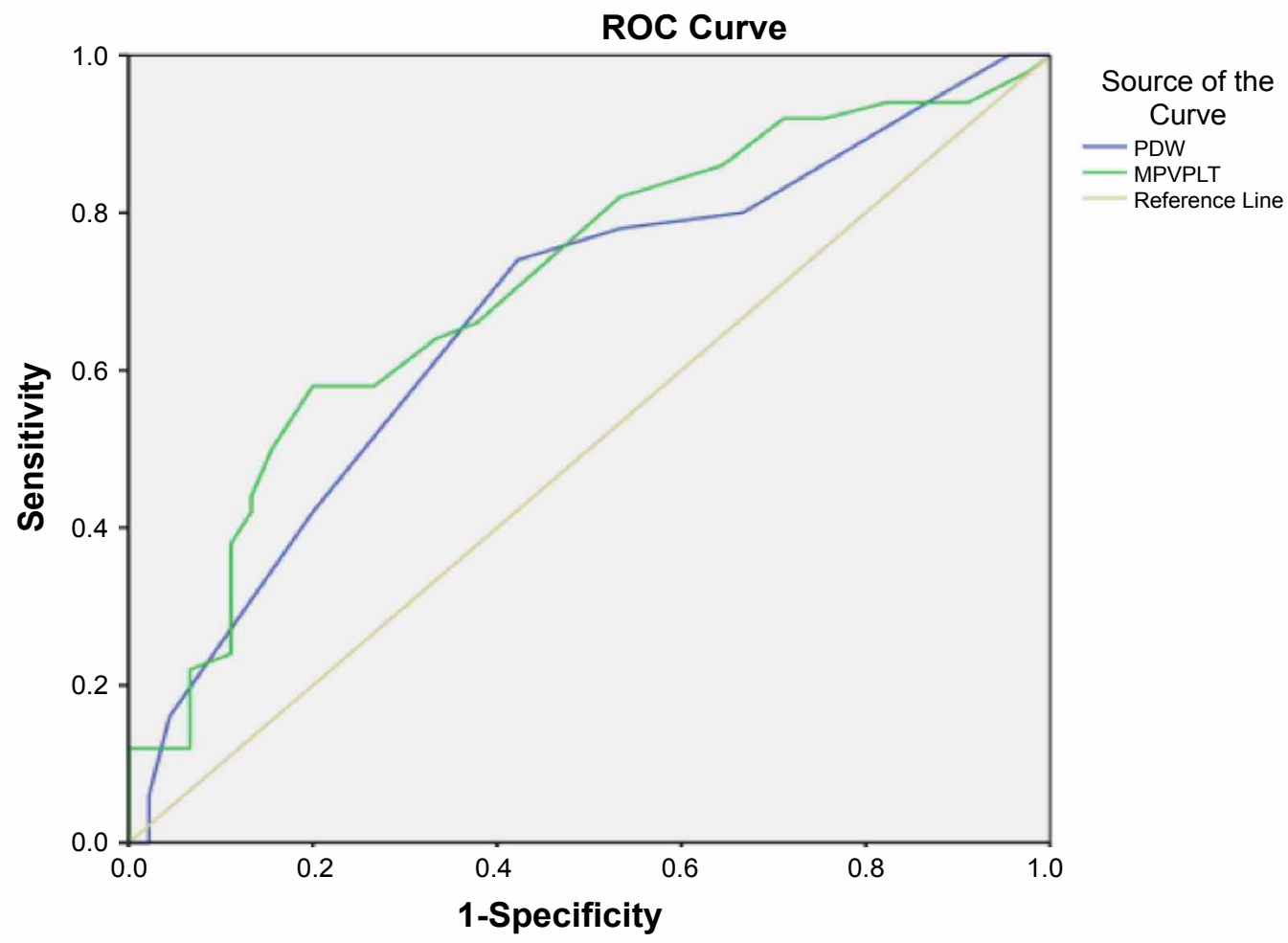

Figure 4: In ROC curve analyses, a PDW and MPV/PLT values of 18.50 and 0.027 were determined as an effective cut-off point in coronary artery ectasia with a sensitivity of $74 \%, 66 \%$ and a specificity of $58 \%, 63 \%$, respectively. (AUC $=0.63$; $p=0.02 ; 95 \% \mathrm{Cl}(0.52-0.74)$. (PDW; $\mathrm{AUC}=0.67 ; \mathrm{p}=0.04 ; 95 \% \mathrm{Cl}(0.56-0.78), \mathrm{MPV} / \mathrm{PLT} ; \mathrm{AUC}=0.71 ; \mathrm{p}=0.01 ; 95 \% \mathrm{Cl}$ $(0.60-0.81))$.

rate among these patients. Moreover, MPV/PLT ratio was significantly higher in patients with stroke after acute myocardial infarction (AMI) [19]. This finding indicated that the MPV/PLT ratio was related to stroke in patients with AMI. In this study, we found that the MPV/PLT ratio was higher in CAE patients, and there was a significant correlation between MPV/PLT ratio and CAE.

Platelet activation is related to inflammation and thrombosis, and MPV is used to measure platelet size [20]. The MPV is an indicator of platelet activation and plays a role in CAD pathophysiology and endothelial dysfunction [21,22]. Recent studies have demonstrated that MPV is increased in acute myocardial infarction and unstable angina pectoris $[23,24]$. In acute myocardial infarction, acute ischemic stroke, preeclampsia, and acute renal stenosis have exhibited increased MPV levels. Also, MPV levels are associated with the poor prognosis in acute thrombotic events. Balta, et al. evaluated the relationship between coronary artery ectasia and neutrophil-lymphocyte (N/L) ratio, and they found that the $\mathrm{N} / \mathrm{L}$ ratio and MPV were significantly increased in patients with CAD and CAE in comparison the control participants [25]. In another study by Yilmaz, et al. have documented that the MPV values are significantly increased during the exercise stress test in patients with significant CAD, compared with the subjects with non-significant CAD [26]. It has also been reported that MPV is increased in patients with CAE, compared to controls with a normal coronary angiogram [27]. Ozbek, et al. investigated the association between MPV and severity of CAE [28]. They indicated that MPV values were significantly higher in the CAE group than the control group, and the results from regression analysis indicated that MPV was an independent predictor of CAE. Also, in the same study, the authors showed that both MPV and red blood cell distribution width (RDW) increased with CAE and MPV was related to CAE severity. Demir, et al. [29] MPV was the highest in subgroup type-I individuals with coronary artery ectasia, although this was not found by Sen, et al. who presented that increased MPV was associated with the atherosclerotic process rather than the severity of the ectasia [30]. In our investigation, we could not find any relationship between MPV and CAE severity. Moghadam, et al. evaluated 14 studies, related to MPV and CAE, in a, and they showed that MPV values were significantly higher in CAE groups. Similarly, we found statistically insignificant increased MPV values in patients with CAE compared to healthy subjects. It is possible that increased MPV values may display the altered platelet reactivity and aggregation in patients with isolated CAE.

The histopathological characteristics of CAE are similar to coronary atherosclerosis, and the mechanism of abnormal luminal dilatation is not well known. In histologic evaluation, ectatic segments 
show atherosclerotic changes and degeneration of the media layer of vascular walls. Also, it is suggesting that a coronary ectasia is a form of CAD due to more than $50 \%$ of patients with ectasia show comorbidity with coronary atherosclerosis. The hypothesis for the etiopathogenesis of CAE is associated with vascular endothelial dysfunction and inflammation [31]. Vascular inflammatory in CAE is presented as elevated inflammatory cytokines and leukocyte count. A previous study showed that serum IL-6 levels were significantly higher in patients with CAE compared to control subjects [32]. Turhan, et al. showed the increased serum $C$-reactive protein levels in patients with isolated CAE compared to patients with obstructive CAD without CAE and normal patients. Similarly Bitigen, et al. found that the CRP levels were significantly higher in patients with isolated CAE compared to controls [27]. High levels of CRP play an essential role in the pathogenesis of $\mathrm{CAE}$, and it may also contribute to the formation of thrombosis in CAE. Also, patients with isolated CAE have elevated levels of plasma soluble intercellular adhesion molecule-1, vascular cell adhesion molecule-1, and E-selectin compared to patients with obstructive CAD without CAE and control subjects with normal coronary arteries [33].

Markis, et al. have presented a classification for $\mathrm{CAE}$, and they showed the increased mortality rates in patients with CAE [4]. Exercise-induced myocardial ischemia has been described in patients with isolated $\mathrm{CAE}$, and some studies suggested that impaired epicardial flow pattern as a possible cause of coronary insufficiency [34]. When the coronary blood flow velocity decreases, the blood viscosity increases so that it may lead to activation of platelets and the coagulation system. In one study, coronary microvascular perfusion was analyzed in patients with isolated CAE with Thrombolysis in Myocardial Infarction (TIMI) flow grades and myocardial blush grades method and reported that TIMI flow grades and myocardial blush grades were lower compared to the non-ectatic arteries of the same patients or controls [9]. These finding is suggesting an impaired microvascular and epicardial perfusion in patients with CAE. A previous study has also been shown that the coronary flow reserves were significantly lower in patients with $C A E$ [10]. Also, Coronary ectasia disease increases mortality rates with including slow coronary flow, coronary vasospasm, dissection, and thrombus formation [30].

\section{Limitations}

This present study has some limitations. This was a single-center study and based on a relatively small group of patients. We ruled out significant coronary artery disease with coronary angiography and determined the stenosis degree by eye. The use of the fractional flow reserve would probably be beneficial for ruling out significant CAD. Lack of other establis- hed inflammatory markers, such as interleukin- 6 and tumor necrosis factor- $\alpha$, is another limitation of the study. The MPV/PLT ratio was measured only once on admission, and we could not measure the changes in the MPV/PLT ratio in response to medical treatment. Further studies on a larger patient population are needed to detect the relationship between MPV/ $\mathrm{PLT}$ ratio and CAE.

\section{Conclusion}

In conclusion, to the best of our knowledge, this is the first study showing that the MPV/PLT ratio is significantly associated with CAE. The present study demonstrates that MPV/PLT ratio is significantly higher in patients with isolated CAE when compared to controls with normal coronary artery, and MPV/PLT ratio is significantly correlated with the presence of CAE. These findings are suggesting that platelets, as well as inflammatory and thrombotic activities, play a role in CAE disease.

\section{Disclosure Statement}

All authors have no declarations of interest to report.

\section{Contributor's Statement}

R.A, E.A performed the research and analysed data.

E.A, R.A provided clinical data.

R.A, E.A. wrote the paper and all authors critically reviewed and edited the paper.

\section{References}

1. Ross R (1999) Atherosclerosis--an inflammatory disease. N Engl J Med 340: 115-126.

2. Libby $P(2003)$ Vascular biology of atherosclerosis: Overview and state of the art. Am J Cardiol 91: 3A-6A.

3. Boles U, Johansson A, Wiklund U, Sharif Z, David S, et al. (2018) Cytokine disturbances in coronary artery ectasia do not support atherosclerosis pathogenesis. Int J Mol Sci 19.

4. Markis JE, Joffe CD, Cohn PF, Feen DJ, Herman MV, et al. (1976) Clinical significance of coronary arterial ectasia. Am J Cardiol 37: 217-222.

5. Befeler B, Aranda JM, Embi A, Mullin FL, El-Sherif N, et al. (1977) Coronary artery aneurysms: Study of their etiology, clinical course and effect on left ventricular function and prognosis. Am J Med 62: 597-607.

6. Turhan H, Erbay AR, Yasar AS, Balci M, Bicer A, et al. (2004) Comparison of C-reactive protein levels in patients with coronary artery ectasia versus patients with obstructive coronary artery disease. Am J Cardiol 94: 1303-1306.

7. Wang $Y$, Wang $Y$, Yin D, Dou K, Wu Y, et al. (2017) The beneficial effects of renin-angiotensin system blockades on 2 year outcomes in coronary artery ectasia patients. Curr Med Res Opin 33: 1677-1684.

8. Yalcin AA, Akturk IF, Celik O, Erturk M, Hancer VS, et al. (2014) Coronary artery ectasia is associated with the c. 894G > T (Glu298Asp) polymorphism of the endothelial nitric oxide synthase gene. Tohoku J Exp Med 232: 137-144. 
9. Gulec S, Atmaca Y, Kilickap M, Akyürek O, Aras O, et al. (2003) Angiographic assessment of myocardial perfusion in patients with isolated coronary artery ectasia. Am J Cardiol 91: 996-999.

10. Akyürek Ö, Berkalp B, Sayın T, Kumbasar D, Kervancıoğlu C, et al. (2003) Altered coronary flow properties in diffuse coronary artery ectasia. Am Heart J 145: 66-72.

11. Gziut AI, Gil RJ (2008) Coronary aneurysms. Pol Arch Med Wewn 118: 741-746.

12. Gasparyan AY, Ayvazyan L, Mikhailidis DP, Kitas GD (2011) Mean platelet volume: A link between thrombosis and inflammation? Curr Pharm Des 17: 47-58.

13. Lai H, Xu R, Yang Y, Ma Y, Li X, et al. (2015) Association of mean platelet volume with angiographic thrombus burden and short-term mortality in patients with ST-segment elevation myocardial infarction undergoing primary percutaneous coronary intervention. Catheter Cardiovasc Interv 85: 724-733.

14. Cameron H, Phillips R, Ibbotson R, Carson P (1983) Platelet size in myocardial infarction. Br Med $\mathrm{J}$ (Clin Res Ed) 287: 449-451.

15. Azab B, Torbey E, Singh J, Akerman M, Khoueiry G, et al. (2011) Mean platelet volume/platelet count ratio as a predictor of long-term mortality after non-ST-elevation myocardial infarction. Platelets 22: 557-566.

16. Schiller NB, Shah PM, Crawford M, DeMaria A, Devereux $R$, et al. (1989) Recommendations for quantitation of the left ventricle by two-dimensional echocardiography. American society of echocardiography committee on standards, subcommittee on quantitation of two-dimensional echocardiograms. J Am Soc Echocardiogr 2: 358-367.

17. Hartnell G, Parnell B, Pridie R (1985) Coronary artery ectasia. Its prevalence and clinical significance in 4993 patients. Br Heart J 54: 392-395.

18. Bolat I, Akgul O, Cakmak HA, Pusuroglu H, Somuncu U, et al. (2016) The prognostic value of admission mean platelet volume to platelet count ratio in patients with ST-segment elevation myocardial infarction undergoing primary percutaneous coronary intervention. Kardiol Pol 74: 346-355.

19. Guenancia C, Hachet O, Stamboul K, Béjot Y, Leclercq T, et al. (2017) Incremental predictive value of mean platelet volume/platelet count ratio in in-hospital stroke after acute myocardial infarction. Platelets 28: 54-59.

20. Wang RT, Li Y, Zhu XY, Zhang Y (2011) Increased mean platelet volume is associated with arterial stiffness. Platelets 22: 447-451.
21. Sen N, Basar N, Maden O, Ozcan F, Ozlu MF, et al. (2009) Increased mean platelet volume in patients with slow coronary flow. Platelets 20: 23-28.

22. Demirkol S, Balta S, Unlu M, Yuksel UC, Celik T, et al. (2012) Evaluation of the mean platelet volume in patients with cardiac syndrome X. Clinics (Sao Paulo) 67: 10191022.

23. Mathur A, Robinson MS, Cotton J, Martin JF, Erusalimsky JD (2001) Platelet reactivity in acute coronary syndromes: Evidence for differences in platelet behaviour between unstable angina and myocardial infarction. Thromb Haemost 85: 989-994.

24. Pizzulli L, Yang A, Martin J, Lüderitz B (1998) Changes in platelet size and count in unstable angina compared to stable angina or non-cardiac chest pain. Eur Heart J 19: 80-84.

25. Balta S, Demirkol S, Celik T, Kucuk U, Unlu M, et al. (2013) Association between coronary artery ectasia and neutrophil-lymphocyte ratio. Angiology 64: 627-632.

26. Yilmaz MB, Saricam E, Biyikoglu SF, Guray Y, Guray U, et al. (2004) Mean platelet volume and exercise stress test. J Thromb Thrombolysis 17: 115-120.

27. Bitigen A, Tanalp AC, Elonu OH, Karavelioglu Y, Ozdemir $\mathrm{N}$ (2007) Mean platelet volume in patients with isolated coronary artery ectasia. J Thromb Thrombolysis 24: 99-103.

28. Özbek K, Katlandur H, Keser A, Ulucan Ş, Özdil H, et al. (2016) Is there a relationship between mean platelet volume and the severity of coronary ectasia? Biomedical Research 27.

29. Demir S, Avsar MK, Karakaya Z, Selcuk M (2013) Increased mean platelet volume is associated with coronary artery ectasia. Postępy Kardiol Interwencyjnej 9: 241-245.

30. Sen N, Tavil Y, Yazici HU, Hizal F, Açikgöz SK, et al. (2007) Mean platelet volume in patients with coronary artery ectasia. Med Sci Monit 13: 356-359.

31. Lind L (2003) Circulating markers of inflammation and atherosclerosis. Atherosclerosis 169: 203-214.

32. Tokgozoglu L, Ergene O, Kinay O, Nazli C, Hascelik G, et al. (2004) Plasma interleukin-6 levels are increased in coronary artery ectasia. Acta Cardiol 59: 515-519.

33. Yilmaz H, Tayyareci G, Sayar N, Gurkan U, Tangurek B, et al. (2006) Plasma soluble adhesion molecule levels in coronary artery ectasia. Cardiology 105: 176-181.

34. Krüger D, Stierle U, Herrmann G, Simon R, Sheikhzadeh A (1999) Exercise-induced myocardial ischemia in isolated coronary artery ectasias and aneurysms ("dilated coronaropathy"). J Am Coll Cardiol 34: 1461-1470. 